\title{
La elección de la lengua extranjera como titulación universitaria: Expectativas y rasgos de estudio
}

The choice of a foreign language as university degree: Expectations and traits of study

\author{
Martínez-Martínez, Asunción ${ }^{(1)}$; Zurita-Ortega, Félix ${ }^{(1)}$; Castro-Sánchez, Manuel ${ }^{(2)}$; Espejo- \\ Garcés, Tamara $^{(3)}$; Chacón-Cuberos, Ramón ${ }^{(4)}$ \& Ruiz-Rico-Ruiz Gerardo ${ }^{(2)}$ \\ (1) Universidad de Granada; (2) Universidad de Almería; (3) Universidad de Cádiz; (4) Universidad de Huelva
}

\begin{abstract}
The aim of this study is to report the degree which is most demanded by students in their final year of university and training Higher Level and to analyze the influence of parameters of gender, field of study, academic and professional interests and motives in the choosing a degree or qualification Foreign Language. This study involves a total of 1,162 students from Spain, with an age between 17 and 21 occurs years $(M=18.24$ years, $S D=1.258)$. The main conclusions of this research is drawn that about one in ten are students who intend to study the three degrees of a foreign language is in the University (Grade of English Studies, Translation and Interpretation and Primary Mention Foreign Language), with women being the ones most Grades intend to study a foreign language, coming almost entirely School, characterized by being altruistic and noting "the taste for this profession," as most decisive reason.
\end{abstract}

Keywords:

Educational Guidance, Foreign Language, Degrees

Reception

Date

2017 March 22

Approval

Date

2017

November 09

Publication

Date:

2017

November 13

\begin{abstract}
Resumen
El objetivo de este estudio es dictaminar cual es el grado más demandado por alumnos de último curso de Bachillerato y Ciclos Formativos de Grado Superior, así como analizar la influencia que tienen parámetros de género, rama de estudios, intereses académicos profesionales y motivos en la elección de un grado o titulación de Lengua Extranjera. En este estudio participaron un total de 1.162 estudiantes de España, con una edad comprendida entre los 17 y 21 años (M=18,24 años; DT= 1,258). Como principales conclusiones de la presente investigación se extrae que aproximadamente uno de cada diez son los estudiantes que pretenden estudiar los tres grados de Lengua Extranjera que hay en la Universidad (Grado de Estudios Ingleses, Traducción e Interpretación y Primaria Mención en Lengua Extranjera), siendo las mujeres las que tienen más intención de estudiar Grados de Lengua Extranjera, procediendo casi en su totalidad de Bachillerato, caracterizándose por ser altruistas y señalando "el gusto por esta profesión" como motivo más determinante.
\end{abstract}

\section{Palabras clave:}

Orientación Educativa, Lengua Extranjera, Grados
Fecha de recepción 2017 March 22

Fecha de aprobación 2017 Noviembre 09

Fecha de publicación 2017 Noviembre 13
La integración de la Universidad Española en el Espacio Europeo de Educación Superior ha supuesto que el conocimiento alcance una posición elevada en las necesidades de los estudiantes universitarios, destacándose la importancia del dominio de la lengua extranjera. Su valor es percibido por la necesidad para comunicarse alcanzando papeles esenciales en los nuevos paradigmas y contextos sociales actuales (Gómez, Solaz \& Sanjosé, 2014). En este sentido, se considera esencial apreciar y tener presentes los aspectos que conlleva de interculturalidad y sensibilidad cultural y social (Frank, 2000; González, 2015) ya que la concienciación de su relevancia en la actualidad puede promover 
el interés y la preocupación de mejorar los niveles de lengua extranjera en nuestro país.

En las universidades se han diseñado nuevos planes de estudios, buscando principalmente tanto el desarrollo de competencias como el conocimiento de la lengua extranjera, la cual ha alcanzado niveles de tipo prioritario (Graduados en Primaria e Infantil). Resulta imprescindible acreditar dichos conocimientos para la obtención de las titulaciones de grado (Halbach, Lázaro \& Pérez-Guerra, 2013) y asimismo, en otros niveles de enseñanza como el Master en Formación del Profesorado de Secundaria, Bachillerato, Formación Profesional y Enseñanza de Idiomas, como requisito de admisión.

En esta línea, se señala la importancia de conocer las concepciones que tienen los estudiantes respecto al conocimiento de una segunda lengua, pues se destaca el concepto de "Aula Bilingüe" como aquel que obtiene una gran relevancia en la sociedad (FernándezCezar, Aguirre \& Harris, 2013; Galvis, 2015) $\mathrm{y}$, del mismo modo, entender las necesidades formativas que se requieren para los estudiantes de titulaciones superiores, poniendo un mayor énfasis en conocer dichas demandas en estudiantes de Grado (Gómez, Solaz \& Sanjosé, 2014).

La organización de la enseñanza de lenguas extranjeras repercute en dos elementos, por un lado en la labor de los profesores (MartínezLirola, 2007) y por otro, en la estructuración de la enseñanza (Conde, 2010). En este sentido, Braga y Domínguez (2010) señalan la utilidad del idioma extranjero como medio de instrucción en otras asignaturas.

Entre los factores que intervienen en el estudio de una segunda lengua, indican varios autores la presencia de tres grupos de parámetros, siendo uno de ellos el de elementos externos al individuo, como son la relación con el medio, contexto y situación de aprendizaje; los elementos internos, como es la lengua materna, conocimiento del mundo y conocimiento lingüístico; y por último los factores individuales, donde se encuadran la edad, motivación, aptitud, etc. (García-
Sánchez \& Cruz, 2013; Pérez-Puente, 2006; Ramos, 2011).

Atendiendo a ello, resulta de gran interés agrupar los elementos en componentes principales, para saber qué grupos son los que más influyen y tenerlos presentes para la posible implementación en el aula de Secundaria y Bachillerato, consiguiendo repercutir en la elección de esta opción por parte de los alumnos de las enseñanzas medias.

En la sociedad actual es cada vez mayor la preocupación de los sujetos jóvenes en cuanto al devenir futuro se refiere. En el siglo pasado alcanzar los 18 años suponía dos itinerarios claramente diferenciados, por un lado se encontraban aquellos jóvenes que se encaminaban al desarrollo laboral, mientras que por otro estaban aquellos que seguían encauzados a la continuación de su formación, pero esto en la actualidad es una paradoja, propiciado por la cada vez mayor carencia de empleo (Blanco, Mercado \& Prado, 2012; Mingorance-Arnáiz \& Pampillón, 2015; Ruesga, Martín y Pérez, 2010; ViñuelaJiménez, Rubiera-Morollón \& Cueto, 2010). En este sentido, el desempleo generado por una crisis económica a nivel mundial que afecta sobre todo a España, está mostrando tendencias a que los jóvenes se decanten por seguir formándose con estudios superiores a pesar de los aun amargos datos sobre las tasas de abandono escolar.

La orientación educativa, como campo de estudio que se preocupa por el desarrollo del alumnado y el vínculo con el desarrollo de la sociedad, se considera como un elemento clave dentro del contexto de la educación, resultando una herramienta indispensable para ofrecer la información y conocimiento sobre aquello que los alumnos demandan $\mathrm{y}$ pretenden realizar en su futuro a corto y medio plazo. A pesar de la relevancia de este proceso, en ciertas ocasiones la vinculación y coordinación que se lleva a cabo entre los Centros de Secundaria y Bachillerato con los Centros Universitarios no es la más idónea (Cepero, 2009; Domínguez, Álvarez \& López, 2013; Llosa, Acín, Cragnolino \& Del Carmen, 
2012) resultando ser un indicio a tener presente y promover su inclusión real en las universidades.

Para tratar la influencia de los intereses del alumnado en la elección vocacional se debe atender a la conceptualización del término. Hernández-Franco (2004) y MartínezMartínez, Castro, Lucena y Zurita (2015), señalan que los intereses expresados son aquellos que hacen referencia a cuestiones ocupacionales específicas del tipo ¿en qué profesión concreta te gustaría trabajar? o bien ¿Qué carrera universitaria te gustaría estudiar cuando acabes el bachillerato? En esta línea, Martín-Moreno (1996) diferencia entre intereses intrínsecos (vivencial, vocacional y profesional) y extrínsecos (circunstanciales y fácilmente modificables), por lo que se puede apreciar la importancia de tratar dicha temática.

Son numerosos los estudios que han descrito las opciones universitarias de los alumnos, siendo el ámbito del Bachillerato de forma general lo más investigado (Cortés \& Conchado, 2012; Santana, Feliciano \& Jiménez, 2012). En este sentido, en escasas ocasiones se han realizado estudios bajo el punto de vista de las lenguas, se puede destacar el de Saravia y Bernaus (2008), sobre la docencia universitaria en alumnos de Ciencias de la Salud y de la Educación, o trabajos previos como los de Norris (2001) o Lasagabaster (2003) sobre motivaciones hacia el estudio de Lenguas Extranjeras en la Universidad.

Asimismo, Cepero (2009) realiza un estudio sobre el contexto vocacional donde las cualidades percibidas por uno mismo influyen en la elección universitaria; Lorente, Calvo, Redondo, Gallego, Benedito y Cano (2011) señalan la importancia de la posterior salida profesional tras la realización de la titulación como decisivo para dicha elección. En este sentido, Gallo, González y Salinero (2010) y Lagares, Ordaz y Lagares (2012) exponen sobre la motivación de elección, considerándose la motivación por el trabajo, el compañerismo, la remuneración y el horario como elementos más motivadores. Por último en esta línea, Lorenzo-Moledo, Argos, Hernández y Vera (2014) citan las formas de acceso a la Universidad teniendo en cuenta las diversas opciones y sus limitaciones como marco conocedor del perfil del alumnado; son algunos de los estudios encaminados a dar respuesta a las posibilidades de éxito y limitaciones de los estudiantes, planteando un elemento esencial que es la conexión entre los estudios secundarios postobligatorios y los universitarios.

Dada la labor profesional ejercida desde la Facultad de Ciencias de la Educación, se considera importante verificar la cantidad de estudiantes de último curso de Bachillerato y Ciclos Formativos que pretenden estudiar un Grado relacionado con la Lengua Extranjera (Grado de Primaria, Traductores o Filología Inglesa) y dictaminar si la decisión viene propiciada por parámetros de género, académicos o de expectativas laborales.

Por lo que el presente estudio pretende aportar cifras sobre lo que acontece en sujetos de 17 a 21 años (M=18,24 años) en relación a la decisión académica de su futuro inmediato, planteándose como objetivos los siguientes:

- Determinar las titulaciones más demandadas en alumnos de último curso de Bachillerato y Ciclos Formativos, así como describir las características académicas y sociodemográficos de los mismos.

- Conocer el número de estudiantes que pretenden acceder a una titulación relacionada con Lengua Extranjera.

- Analizar la influencia que tienen parámetros de género, rama de estudios, intereses académicos profesionales $\mathrm{y}$ motivos en la elección de un grado o titulación de Lengua Extranjera.

\section{Método}

En este estudio se utilizó un diseño de carácter cuantitativo descriptivo y de tipo transversal. Posteriormente se desarrolló un estudio relacional para analizar el grado de 
dependencia entre las distintas variables objeto de estudio.

\section{Muestra}

Los participantes se generan del total de una población de 6.132 alumnos/as matriculados en último curso de Bachillerato y Ciclos Formativos de Granada (España), según datos facilitados por el Ministerio de Educación durante el curso académico 2012/2013; para establecer una muestra representativa (error al 0,02 ; I.C $=95,5 \%$ ) se emplearon técnicas de estratificación y proporcionalidad, considerándose tipo de rama académica (ciclos formativos y bachillerato) y sexo (hombresmujeres). Participaron en esta investigación una muestra total de 1.162 estudiantes de España, con una edad comprendida entre los 17 y 21 años (M=18,24 años; DT= 1,258) y que presentaron errores muestrales del 0,02 en el total de la muestra y por estratos del 0,03 para Bachillerato y 0,04 para Ciclos Formativos, analizándose un total del 18,98\% del universo total. El estudio cumplió con las normas éticas del Comité de Investigación y Declaración de Helsinki de 1975. Se contó en todos los casos con el consentimiento informado de los participantes y se respetó el resguardo a la confidencialidad.

\section{Instrumentos}

La presente investigación tomó como referencia las siguientes variables e instrumentos de medida:

- Formación, categorizado en Bachillerato y Ciclos Formativos.

- Género, según masculino o femenino.

- Titulación de Elección por Rama de Conocimiento, en función de lo planteado por Martínez-Martínez (2013) se desarrolla el ámbito de conocimiento de la titulación universitaria a elegir en el próximo curso planteándose las siguientes especialidades (Arqueología, Arquitectura, Audición y Lenguaje, Bellas Artes, Biblioteconomía, Biología, Ciencias Ambientales, Ciencias del Trabajo, Ciencias Políticas, Comunicación Audiovisual, Criminología, Derecho, Económicas, Empresariales,
Enfermería, Estudios Ingleses, Farmacia, Filología, Filosofía, Física-Química, Fisioterapia, INEF, Informática, Ingeniería, Literatura, Magisterio, Matemáticas, Medicina, Militar, Música, Odontología, Óptica, Pedagogía, Periodismo, Psicología, Relaciones Laborales, Trabajo Social, Traductores, Turismo, Veterinaria y Otras). Quedando categorizadas por Ramas del Conocimiento.

- Especialidad en Lengua Extranjera, para ello se les planteó la cuestión, ¿Deseas estudiar una titulación de Lengua Extranjera?, incluyendo para ello una respuesta de tipo dicotómico, Si o No.

- Expectativas y características de la elección, para se utilizó el cuestionario de Intereses Académicos Profesionales (CIBAP) de Hernández-Franco (2004), concretamente en lo concerniente al grado de expectativas y valores entendidos como grado de preferencia hacía unos determinados parámetros planteados por Bandura (1987). Este instrumento consta de 11 cuestiones descritas a partir de una serie de afirmaciones, determinados a través de una escala Likert de cinco opciones (1= Total Desacuerdo; 2= En Desacuerdo; 3=Término Medio; 4= De Acuerdo y 5= Total Acuerdo). Los ítems de esta escala se agrupan en cuatro constructos denominados por Hernández-Franco (2004), en: Altruismo (I.3-I.6-I.8); Seguridad (I.2-I.5I.11); Prestigio-Poder (I.1-I.9); Hedonismo (I.4-I.7-I.10). Señalar que el alfa de Cronbach para este cuestionario determinó una alta fiabilidad $(\alpha=0,858)$.

- Motivo más Determinante, valorándose cuál de los motivos es el más determinante para la elección de la preinscripción según la a) Facilidad, b) Titulación básica y flexible, c) Salir de casa, d) Permanecer en casa, e) Perspectiva de empleo, f) Perspectiva de buenos salarios, g) Gusto por esta profesión, h) Concordar con mis cualidades, i) Ocupación con futuro, j) Insistencia paterna, K) Disponer de otro título y l) Otra. 


\section{Procedimiento}

En primer lugar, a través de la Facultad de Ciencias de la Educación, y en contacto con la Consejería de Educación de la Junta de Andalucía, se solicitó la colaboración de los Centros Educativos seleccionados, a partir de un muestreo de conveniencia de las categorías objeto de estudio. La dirección de cada Centro Educativo fue informada sobre la naturaleza de la investigación y solicitando la colaboración de sus alumnos/as. En segundo lugar se adjuntó un modelo de autorización destinado a los responsables legales de los adolescentes pidiéndoles su consentimiento informado, en el caso de que fuesen menores de edad.

En todo momento se garantizó a los participantes el anonimato de la información recogida aclarando que su utilización sería sólo con fines científicos. Los encuestadores estuvieron presentes durante la recogida de los datos para poder resolver cualquier duda al respecto. Esta se desarrolló sin ningún tipo de problema o anormalidad a reseñar. En último lugar se agradeció a los docentes, orientadores y responsables su colaboración y se les informó del envío en un futuro próximo de un informe sobre los datos obtenidos respetando la confidencialidad de los mismos.

Para el análisis de los datos se utilizó el programa estadístico software SPSS 22.0., mediante la utilización de descriptivos, medias y desviación típica se desarrolló la primera parte del análisis encaminado a determinar el perfil de los participantes, en segundo lugar se estableció el análisis comparativo para lo cual se emplearon tablas de contingencia para establecer las diferencias entre las distintas variables objeto de estudio.

\section{Resultados}

En este trabajo de investigación realizado sobre 1.162 participantes, se constató que el $41,9 \% \quad(n=487)$ eran varones y el $58,1 \%$ $(n=675)$ mujeres, la mayoría estaban estudiando Bachillerato (58,6\%; $n=682$ ), y los restantes $480 \quad(41,4 \%)$ cursaban Ciclos Formativos de Grado Superior. Con respecto a las titulaciones o grados que los estudiantes quieren cursar y descartando al 17,0\% $(n=196)$ que no querían seguir estudiando, las tres especialidades universitarias con mayor demanda son por orden de preferencia: Magisterio (12,5\%; $n=145)$ perteneciente a la rama de Ciencias Sociales y Jurídicas siendo también la más demandada con un porcentaje de $32,9 \%(n=382)$, Ingeniería $(7,6 \% ; n=88)$ perteneciente a la rama de Ingenierías y Arquitecturas con un total de $12,4 \%(n=144)$ y Enfermería (6,4\%; $n=75)$ concerniente a la rama de Ciencias de la Salud que alcanza un 21,7\% (n=254) Tabla I.

Tabla 1. Descriptivos sobre la intención de estudio.

\begin{tabular}{|c|c|c|c|}
\hline \multicolumn{4}{|l|}{ SEXO } \\
\hline \multicolumn{4}{|c|}{$41.9 \%(n=487)$} \\
\hline Femenino & \multicolumn{2}{|c|}{$58.1 \%(\mathrm{n}=675)$} & \\
\hline \multicolumn{2}{|c|}{$\begin{array}{l}\text { TITULACIONES POR RAMA } \\
\text { DE CONOCIMIENTO }\end{array}$} & \multicolumn{2}{|l|}{$\begin{array}{l}\text { INTENCIÓN MÁS } \\
\text { DETERMINANTE }\end{array}$} \\
\hline No Estudiar & $17 \%(n=196)$ & Facilidad & $3,1 \%(n=35)$ \\
\hline Ciencias Sociales y Jurídicas & $32.9 \%(n=382)$ & Titulación básica y flexible & $0,6 \%(n=7)$ \\
\hline Ciencias de la Salud & $21.7 \%(n=254)$ & Salir de casa & $3,3 \%(n=37)$ \\
\hline Arte y Humanidades & $5 \%(n=59)$ & Permanecer en casa & $1,8 \%(n=20)$ \\
\hline Ciencias & $4.5 \%(n=52)$ & Perspectivas de empleo & $12,5 \%(n=145)$ \\
\hline Ingenierías y Arquitectura & $12.4 \%(n=144)$ & Perspectivas de buenos salarios & $9,4 \%(n=109)$ \\
\hline \multirow[t]{5}{*}{ Otras } & $6,6 \%(n=75)$ & Gusto por esta profesión & $47,4 \%(n=552)$ \\
\hline & & Porque es una ocupación con futuro & $5,6 \%(n=65)$ \\
\hline & & Insistencia paterna & $1,8 \%(n=21)$ \\
\hline & & Disponer de un título & $5,0 \%(n=58)$ \\
\hline & & Otra & $1,9 \%(n=22)$ \\
\hline
\end{tabular}

* La opción de Otras, todas aquellas titulaciones con menos de 10 aportaciones. 
Tabla 2. Descriptivos las expectativas de área vocacional de interés preferente.

\begin{tabular}{|c|c|}
\hline \multicolumn{2}{|c|}{$\begin{array}{l}\text { EXPECTATIVAS DE ÁREA } \\
\text { VOCACIONAL INTERÉS PREFERENTE }\end{array}$} \\
\hline $\begin{array}{l}\text { Altruismo } \\
\end{array}$ & $35.1 \%(n=408)$ \\
\hline Seguridad & $33.3 \%(n=388)$ \\
\hline Prestigio-Poder & $10.9 \%(\mathrm{n}=125)$ \\
\hline Hedonismo & $20.7 \%(\mathrm{n}=241)$ \\
\hline
\end{tabular}

* La opción de Otras, todas aquellas titulaciones con menos de 10 aportaciones.

Continuando con la descripción de los participantes en cuanto a las características y perspectivas de la elección, en este sentido la mayoría de los estudiantes se postularon como Altruistas (35,1\%; $\mathrm{n}=408)$ y Seguridad $(33,3 \% ; n=388)$. En referencia al motivo más determinante, el 47,4\% $(n=552)$ de los estudiantes señalaron el "Gusto por esta profesión" (Tabla I).

Asimismo, se señaló que los estudiantes que pretenden estudiar titulaciones donde se desarrolla la Lengua Extranjera alcanzan un $6,6 \%(n=77)$, se encuentran encuadrados en tres grados (Filología Inglesa, Traducción e
Interpretación y Primaria Mención en Lengua Extranjera).

Atendiendo a estos resultados, se llevó a cabo un estudio descriptivo centrado en los estudiantes que pretendían estudiar una titulación o grado que incluye la Lengua Extranjera (Tabla II), señalándose que las chicas tienen mayor intención en un $77,9 \%$ $(n=60)$ que los chicos con un 22,1\% $(n=17)$. Proceden principalmente de Bachillerato $(71,4 \% ; n=55)$, se caracterizan por ser personas altruistas $(50,6 \% ; n=39)$ y señalan como opción más determinante "el gusto por esta profesión” en un 45,5\% (n=35).

Tabla 3. Descriptivo del alumnado que tiene intención de estudiar Lengua Extranjera.

\begin{tabular}{lclc}
\hline \multicolumn{2}{c}{ SEXO } & \multicolumn{2}{l}{ INTENCIÓN MÁS DETERMINANTE } \\
\hline Masculino & $22.1 \%(\mathrm{n}=17)$ & Facilidad & $7.8 \%(\mathrm{n}=6)$ \\
\hline Femenino & $77.9 \%(\mathrm{n}=60)$ & Titulación básica y flexible & $0 \%(\mathrm{n}=0)$ \\
\hline \multicolumn{2}{c}{ FORMACIÓN } & Salir de casa & $5.2 \%(\mathrm{n}=4)$ \\
\hline \multicolumn{2}{c}{} & Permanecer en casa & $1.3 \%(\mathrm{n}=1)$ \\
\hline Bachillerato & $71.4 \%(\mathrm{n}=55)$ & Perspectivas de empleo & $13 \%(\mathrm{n}=10)$ \\
\hline Ciclos Formativos & $28.6 \%(\mathrm{n}=22)$ & Perspectivas de buenos salarios & $5.2 \%(\mathrm{n}=4)$ \\
\hline EXPECTATIVAS ÁREA VOCACIONAL & Gusto por esta profesión & $45.5 \%(\mathrm{n}=35)$ \\
\hline \multicolumn{5}{c}{} & Concordar con mis cualidades & $7.8 \%(\mathrm{n}=6)$ \\
\hline Altruismo & $50.6 \%(\mathrm{n}=39)$ & Porque es una ocupación con futuro & $9.1 \%(\mathrm{n}=7)$ \\
\hline Seguridad & $27.3 \%(\mathrm{n}=21)$ & Insistencia paterna & $0 \%(\mathrm{n}=0)$ \\
\hline Prestigio-Poder & $6.5 \%(\mathrm{n}=5)$ & Disponer de un título & $5.2 \%(\mathrm{n}=4)$ \\
\hline Hedonismo & $15.6 \%(\mathrm{n}=12)$ & Otra & $0 \%(\mathrm{n}=0)$ \\
\hline
\end{tabular}


En cuanto al estudio relacional, se pretende establecer el perfil del alumnado que tiene la intención de estudiar un Grado de Lengua Extranjera según el Grado o Titulación de elección. En este sentido, en relación al género no se determinaron diferencias estadísticamente significativas $(p=0,055)$ y de la misma manera con el motivo más determinante $(p=0,386)$.

En esta línea, sí se establecieron diferencias estadísticamente significativas $\left(p \leq .05^{*}\right)$ en relación al curso y expectativas de área vocacional (Tabla III). En el primer caso, los resultados arrojaron que el alumnado de Ciclos Formativos y Bachillerato en proporciones similares $(41,9 \%$ y $58,1 \%$, respectivamente) pretenden estudiar el Grado de Maestro (Mención Lengua Extranjera). Asimismo los datos deparan que en las otras dos titulaciones, Filología y Traductores, la muestra procede en un $82,6 \%$ y en el $100 \%$ de los casos, respectivamente, del Bachillerato.

Tabla 4. Distribución de titulación según curso de procedencia y expectativas de área vocacional

\begin{tabular}{cccccc} 
& & \multicolumn{4}{c}{ ELECCIÓN TITULACIÓN } \\
\cline { 3 - 6 } & & Magisterio & Traductores & $\begin{array}{c}\text { Estudios } \\
\text { Ingleses }\end{array}$ & Sig. \\
\hline CURSO & $\begin{array}{c}\text { Ciclos } \\
\text { Formativos }\end{array}$ & $41.9 \%(\mathrm{n}=18)$ & $0 \%(\mathrm{n}=0)$ & $17.4 \%(\mathrm{n}=4)$ & 0.009 \\
\hline & Bachillerato & $58,1 \%(\mathrm{n}=25)$ & $100 \%(\mathrm{n}=11)$ & $82.6 \%(\mathrm{n}=19)$ & \\
\hline $\begin{array}{c}\text { EXPECTATIVAS } \\
\text { ÁREA } \\
\text { VOCACIONAL }\end{array}$ & Altruismo & $67.4 \%(\mathrm{n}=29)$ & $45.4 \%(\mathrm{n}=5)$ & $21.7 \%(\mathrm{n}=5)$ & 0.002 \\
\hline & Seguridad & $14 \%(\mathrm{n}=6)$ & $18.2 \%(\mathrm{n}=2)$ & $56.6 \%(\mathrm{n}=13)$ & \\
\hline & Prestigio-Poder & $7 \%(\mathrm{n}=3)$ & $18.2 \%(\mathrm{n}=2)$ & $0 \%(\mathrm{n}=0)$ & \\
\hline & Hedonismo & $11.6 \%(\mathrm{n}=5)$ & $18.2 \%(\mathrm{n}=2)$ & $21.7 \%(\mathrm{n}=5)$ & \\
\hline
\end{tabular}

Del mismo modo, se destaca en relación a las expectativas de los estudiantes y con el fin de caracterizar el perfil de los mismos, los participantes que pretenden estudiar el Grado de Maestro son más altruista en un 67,4\% $(n=29)$ que el resto de titulaciones; si bien los alumnos que desean estudiar Traductores también se podrían calificar como altruistas pues alcanza la valoración más alta entre las demás categorías. Encuadrándose los estudiantes que quieren realizar Estudios Ingleses por seguridad dentro de las expectativas como opción más valorada en el $56,6 \%$ de los casos $(n=13)$.

\section{Discusión}

En este estudio se apreció que aproximadamente una quinta parte del alumnado de último curso no pretende seguir estudiando titulaciones de nivel superior (estudios universitarios), asimismo las tres especialidades universitarias con mayor demanda son Magisterio, Ingeniería y
Enfermería pertenecientes cada una de ellas a diversas áreas, lo que corrobora que en éstas el aspecto vocacional es esencial a la hora de elegir los estudios superiores (MartínezRodríguez y Carmona, 2010; MartínezMartínez, 2013), pues tanto en Magisterio como en Enfermería el componente vocacional es bastante elevado. En este sentido, no debemos obviar lo planteado por diversos autores como Lora-López (2008) y Martínez-Martínez (2013), cuando indican que titulaciones de índole sanitario, unidas a las ingenierías (Fernández-López y Vaquero, 2006) suelen ser bastante solicitadas por las expectativas futuras que plantean, ya que popularmente han tenido bastante prestigio social.

Aproximadamente uno de cada diez son los estudiantes que pretenden estudiar los tres grados de Lengua Extranjera (Grado de Estudios Ingleses, Traducción e Interpretación y Primaria Mención en Lengua Extranjera). 
Se puede apreciar que el estudio de una segunda lengua es un elemento esencial en los estudios actuales, pero el número de matrículas en estas titulaciones ha disminuido, así en el año 2005 “Traducción $e$ Interpretación” se encontraba entre las cinco titulaciones más solicitadas mientras que en la actualidad se ha producido un descenso en dicho ranking.

La mayoría de los estudiantes se postularon con características de tipo altruistas y de seguridad al igual que en los estudios de Barra (2002), Sadurní y Rostan (2004) y Alonso (2009), hallaron una predisposición a dedicarse a la atención y al servicio de los demás, propiciado por las rasgos de personalidad que se aprecian en el ser humano (Marina \& Bernabéu, 2007; MartínezRodríguez, 2011). Por otro lado, cerca de la mitad de los casos analizados señalaron el “Gusto por esta profesión”, como la intención más determinante para elegir sus estudios universitarios. Estos datos no concuerdan con los valores y cifras hallados en la literatura (Martínez, Gimeno \& Rocabert, 1998; Fernández-Poncela, 2000; León \& Gambara, 2005), donde se señala que las intenciones de los estudiantes van encaminadas a tener un buen sueldo para mantener a la familia y la economía del hogar.

Respecto a los estudiantes que pretendían estudiar una titulación o grado de Lengua Extranjera, se observó que las chicas tienen más intención de estudiar esta modalidad siendo en tres de cada cuatro participantes al igual que manifestaron en sus estudios Uribe, Gutiérrez y Madrid (2008). En esta misma línea, proceden casi en su totalidad de Bachillerato señalándose del mismo modo en otros estudios revisados (Alonso-Tapia, 1999; Dörnyei 2003; Velasco \& Bernaus, 1999), así como es la motivación hacia el estudio y aprendizaje de una segunda lengua. En este sentido, García-Sánchez y Cruz (2013), añaden que los factores señalados son decisivos y que la personalidad adquiere un papel fundamental, coincidiendo con lo mostrado en los resultados de este estudio donde los participantes que desean estudiar
Grados en Lenguas Extranjeras se caracterizan por ser altruistas y señalan "el gusto por esta profesión”. De este modo, podemos entender que estos estudiantes presentan una vocación hacia el estudio de idiomas. De hecho, Arnaiz y Guillén (2013) indican que los estudiantes de Lengua Extranjera presentan niveles de ansiedad bajos en comparación con sus homólogos universitarios, suponiendo un elemento común y satisfactorio hacia el desarrollo de la elección de éstas titulaciones.

En cuanto a la procedencia formativa de los estudiantes, aquellos que tenían intención de matricularse en el grado de Primaria procedían en cifras similares de Ciclos Formativos y Bachillerato, esta situación puede venir motivada porque algunos módulos se encuentran en relación con la animación y los idiomas, alcanzando un conocimiento práctico relacionado con la lengua extranjera y su enseñanza. Por otro lado, los estudiantes que desean cursar Estudios Ingleses y Traductores proceden exclusivamente del Bachillerato, propiciado por la vocación que se tiene hacia estas titulaciones. En este sentido, debemos tener presente que la nota de acceso a estos Grados es superior que la de Maestro, señalándose en estudios de investigación que el alumnado de último curso de Bachillerato presenta calificaciones superiores a los estudiantes de módulos formativos (Prieto-Zurita, 2010; Martínez-Martínez, 2013).

Del mismo modo, se destaca que el alumnado con intención de cursar los Grados de Maestro y Traducción e Interpretación están caracterizadas por ser altruistas con elementos psicopedagógicos que potencian un aprendizaje significativo (Burillo et al., 2006) y destacando en ellos, la labor de hacer hacia los demás (enseñar) con la predisposición a dedicarse a la atención y al servicio de la comunidad (Barra, 2002; Sadurní \& Rostan 2004).

Para finalizar debemos señalar que se han dado una serie de limitaciones en la realización del presente estudio, producidas 
en su mayoría porque una parte considerable de los estudiantes de Ciclos Formativos rehusaron de la realización de este estudio. Del mismo modo, se podrían haber incluido cuestiones de tipo familiar así como elementos para poder predecir el nivel de inglés que tiene el alumnado que pretende acceder a Grados de Lengua Extranjera, con el fin de ajustar el perfil de estos estudiantes.

Como principales conclusiones de este estudio se extraen las siguientes:

- Aproximadamente uno de cada diez estudiantes que pretenden estudiar los tres grados de Lengua Extranjera que se ofrecen en la Universidad española (Grado de Estudios Ingleses, Traducción e Interpretación y Primaria Mención en Lengua Extranjera).

- Las mujeres tienen más intención de estudiar Grados de Lengua Extranjera, procediendo casi en su totalidad de Bachillerato, caracterizándose por ser altruistas y señalando "el gusto por esta profesión” como motivo más determinante.

\section{Referencias}

Alonso, A. (2009). Orientación no sexista. En: VI Jornadas de Coeducación "Rompiendo Estereotipos" Granada: Centro de Educación del Profesorado de Granada (CEP).

Alonso-Tapia, J. (1999) Motivación y aprendizaje en la Enseñanza Secundaria. En C. Coll. (Coord.) Psicología de la Instrucción: la enseñanza y el aprendizaje en la educación secundaria (pp. 105-108). Barcelona: ICE.

Arnaiz, P., \& Guillen, F. (2013). La ansiedad entre estudiantes españoles de inglés como LE de diferentes titulaciones universitarias. Anales de Psicología, 29(2), 335-344. doi: https://doi.org/10.6018/analesps.29.2.130791

Bandura, A. (1987). Pensamiento y acción: fundamentos sociales. Barcelona: Martínez Roca.

Barra, E. (2002). La influencia del sexo y de la tipificación de Rol sexual. Revista
Latinoamericana de Psicología, (34), 275284.

Blanco, A., Mercado, C., \& Prado, A. (2012). Perfil y motivación de la juventud española emprendedora. Revista de Estudios sobre Juventud, (99), 22-34.

Braga, J., \& Domínguez, E. (2010). Structural Calques: Source Language Interference in CLIL Lectures in Spain. Vienna English Working Papers, 19(3), 5-11. 2010.

Burillo, J., Capdevila, M., Cassany, D., Estaire, S., Holgado, J., López, M. C., López-Boujon, R., Medina, A., Morato, N., Pérez-Esteve, P., Ramos, M., Roig, V., Sánchez, A., Vázquez, E., Vez, J. M., Vila, I. (2006). Las lenguas extranjeras en el aula. Reflexiones y propuestas. Caracas: Editorial Laboratorio Educativo.

Cepero, B. A. (2009). Las preferencias profesionales y vocacionales del alumnado de secundaria y formación profesional específica. Tesis Doctoral: Universidad de Granada.

Conde, A. M. (2010). Innovación y Bolonia: La enseñanza aprendizaje de inglés L3 en la Universidad Pablo de Olavide. ELIA, (10), 167-197.

Cortés, P.A., \& Conchado, A. (2012). Los contextos parentales y académicos y los valores laborales en la toma de decisiones en bachillerato. ESE. Estudios sobre Educación, 22, 93-114.

Domínguez, G., Álvarez, F.J., \& López, A.M. (2013). Acción tutorial y orientación en el periodo de transición de la Educación Secundaria a la Universidad. La orientación en el alumno de nuevo ingreso. REDU, Revista de Docencia Universitaria, 11(2), 221-241. doi: https://doi.org/10.4995/redu.2013.5574

Dörnyei, Z. (2003). Attitudes, Orientations, and Motivation in Language Learning: Advances in Theory, Research and Applications. Language Learning: Journal of Research in Language Studies, 53(1), 332. doi: https://doi.org/10.1111/1467$\underline{9922.53222}$ 
Fernández-Cezar, R., Aguirre, C., \& Harris, C. (2013). La formación de maestros en Aprendizaje Integrado de Contenidos en Lengua Extranjera (AICLE): Un estudio en Castilla La Mancha. Revista de Formación e Innovación Educativa Universitaria, 6(1), 33-44.

Fernández-López, S., \& Vaquero, A. (2006). El grado de adecuación con la oferta a la demanda de titulaciones en el sistema universitario de Galicia. Revista Galega do Ensino, 14(48), 451-470.

Fernández-Poncela, A. (2000). Elecciones, cámaras y mujeres. Barcelona: Servicio de publicaciones de la Universidad Autónoma de Barcelona.

Frank, R. A. (2000). Medical communication: non-native English speaking patients and native English speaking professionals. English for Specific Purposes, 19, 31-62. doi: $\quad$ https://doi.org/10.1016/S08894906(98)00012-X

Galvis, H. A. (2015). Retos para Colombia en el marco del Plan Nacional de Bilingüismo: relato de experiencias in situ. Voces $y$ Silencios: Revista Latinoamericana de Educación, 5(2) 206-218.

Gallo, C., González, C., \& Salinero, J. J. (2010). Estudio comparativo de las motivaciones, percepciones y expectativas de futuro entre estudiantes de CCAFD, de la Universidad Camilo José Cela (España) y la Universidad de Hertfordshire (Inglaterra). Journal of Sport and Health Research, 2(3), 253-260.

García-Sánchez, M.E., \& Cruz, M.L. (2013). Factores motivacionales extrínsecos e intrínsecos en el aula de inglés: análisis empírico. Porta Linguarum, 19, 275-297.

Gómez, A., Solaz, J.J., \& Sanjosé, V. (2014). Competencia en Lengua Inglesa de Estudiantes Universitarios Españoles en el contexto del EEES: nivel de dominio Lingüístico, Estrategias Metacognitivas y Hábitos Lectores. Revista de Educación, 363, 154-183.
González, R. P. (2015). Interculturalidad en clase de Francés Lengua Extranjera Actividades para abrirse al mundo. Campo Abierto, 33(1), 117-136.

Halbach, A., Lázaro, A., \& Pérez-Guerra, J. (2013). La lengua inglesa en la nueva Universidad española. Revista de Educación, 362, 105-132.

Hernández-Franco, V. (2004). Evaluación de los intereses básicos académico profesionales de los estudiantes de secundaria. REOP, 15(1), 117-141.

Lagares, E. C., Ordaz, F. G., \& Lagares, A. R. (2012). Evidencias empíricas en el proceso de elección de los estudios universitarios. Enseñanza y Teaching: Revista interuniversitaria de didáctica, 30, 45-62.

Lasagabaster, D. (2003). Trilingüismo en la enseñanza: Actitudes hacia la lengua minoritaria, la mayoritaria y la extranjera. Lleida: Editorial Milenio.

León, O., \& Gambara, H. (2005). DATUS, Decision Aiding Tool for University Subjects. Herramienta para la elección de asignaturas optativas universitarias. Psicothema, 17(1), 164-168.

Lora-López, P. (2008). Reflexiones sobre el grado y postgrado de Enfermería: la investigación en Enfermería. Index de Enfermería, 17(2), 85-86. doi: https://doi.org/10.4321/S1132$\underline{12962008000200001}$

Lorente, R., Calvo, R., Redondo, A., Gallego, J. R., Benedito, M. A., \& Cano, F. J. (2011). La importancia del conocimiento de las salidas profesionales en la elección y al cursar una titulación. Proyecto mercado laboral de los egresados en relaciones laborales. Proyecto de Innovación educativa del Vicerectorat de Convergència Europea i Qualitat de la Universitat de València.

Lorenzo-Moledo, M., Argos, J., Hernández, J., \& Vera, J. (2014). El acceso y la entrada del estudiante a la universidad: Situación y propuestas de mejora facilitadoras del tránsito. Educación XX1, 17(1), 15-38. 
Llosa, S., Acín, A., Cragnolino, E., \& Del Carmen, M. (2012). La demanda potencial y la demanda efectiva en educación de jóvenes y adultos: primera aproximación al enfoque cuantitativo y cualitativo de la biografía educativa. Reflexiones en torno al significado atribuido a la educación y la capacitación laboral. Cuadernos de Educación, 2, 175-187.

Marina, J.A., \& Bernabéu, R. (2007). Competencia social y ciudadana. Madrid: Alizanza Editorial.

Martínez, B., Gimeno, M. J., \& Rocabert, E. (1998). ¿Existen diferencias en los valores de trabajo buscados por hombres y mujeres de diferente grupo vocacional a la hora de tomar una decisión vocacional? En Actas I Congreso de Orientación y Diversidad. Santiago de Compostela: Tidis-Expoc, S.L.

Martínez-Lirola, M. (2007). El nuevo papel del profesor universitario de lenguas extranjeras en el proceso de convergencia europea y su relación con la interacción, la tutoría y el aprendizaje autónomo. Porta Linguarum, 7, 31-43.

Martínez-Martínez, A. (2013). La orientación como actividad educativa y vocacional en los itinerarios curriculares del alumnado de bachiller y formación Profesional y su inclusión en el Mercado Laboral. Tesis doctoral: Universidad de Granada.

Martínez-Martínez, A., Castro, M., Lucena, M., \& Zurita, F. (2015). Elección de titulación universitaria y expectativas de resultados de los adolescentes de Granada. Revista Española de Orientación $y$ Psicopedagogía, 26(3), 63-77. doi: https://doi.org/10.5944/reop.vol.26.num.3.20 15.16401

Martínez-Rodríguez, F. M., \& Carmona, G. (2010). Test de factores sociopersonales para la inserción laboral de los jóvenes: un instrumento para la evaluación y la formación. ESE. Estudios sobre Educación, 18, 115-138. 2010.

Martínez-Rodríguez, F.M. (2011). Percepción del profesorado de las escuelas taller y casas de oficios en Andalucía acerca del nivel de competencias emprendedoras en su alumnado. Revista de Educación, 356, 303326. 2011.

Martín-Moreno, M.J. (1996). Sistema de Experto de Orientación Vocacional Profesional (Procedimiento Informatizado de ayuda). Tesis Doctoral: Universidad de Madrid.

Mingorance-Arnáiz, A., \& Pampillón, R. (2015). Myths and Realities of the Spanish Labour Market. Estudios de Economía Aplicada, 33, 223-258.

Norris, J. (2001). Motivation as a Contributing Factor in Second Language Acquisition. The Internet TESL Journal, 7(6), 1-8.

Pérez-Puente, E.M. (2006). Las Webquests como elemento de motivación para los alumnos de Educación secundaria obligatoria en la clase de lengua extranjera (inglés). Barcelona: Servicio de Publicaciones de la Universidad de Barcelona.

Prieto-Zurita, M.P. (2010). Estudio de las titulaciones de Magisterio de Educación Física y Educación Infantil en el Centro de Enseñanza superior en Humanidades Ciencias de la Educación "Don Bosco". Tesis Doctoral: Universidad Complutense de Madrid.

Ramos, M.C. (2011). La motivación en la enseñanza del inglés como lengua extranjera. Aula y Docentes, 32, 39-49.

Ruesga, S., Martín, J.L., \& Pérez, L. (2010). Informe de coyuntura sobre el mercado de trabajo en España y Andalucía (Primer trimestre de 2010). Revista de Temas Laborales, 105, 131-159.

Sadurní, M., \& Rostan, C. (2004). La importancia de las emociones en los periodos sensibles del desarrollo. Infancia $y$ aprendizaje, 27(1), 105-114. doi: https://doi.org/10.1174/021037004772902132

Santana, L.E., Feliciano, L., \& Jiménez, A.B. (2012). Toma de decisiones y género en el Bachillerato. Revista de Educación, 359, 357-387. 
Saravia, E., \& Bernaus, M. (2008). Motivaciones y actitudes para el aprendizaje de lenguas de dos colectivos de estudiantes universitarios: futuros maestros de lenguas extranjeras y futuros enfermeros $y$ fisioterapeutas. Porta Linguarum, 10, 163184.

Uribe, D., Gutiérrez, J., \& Madrid, D. (2008). Las actitudes del alumnado hacia el aprendizaje del inglés como idioma extranjero: estudio de una muestra en el sur de España. Porta Linguarum, 10, 85-100.
Velasco, C., \& Bernaus, M. (1999). Los proyectos en el aula de lengua: una herramienta para la motivación. Aula de Innovación Educativa, 87, 39-42.

Viñuela-Jiménez, A., Rubiera-Morollón, F., \& Cueto, B. (2010). An analysis of urban size and territorial location effects on employment probabilities: the Spanish case. Growth and Change. A Journal of Urban and Regional Policy, 41(4), 495-519.

\section{Authors / Autores}

To know more / Saber más

Martínez-Martínez, Asunción (asuncionmm@ugr.es)

Doctora. Departamento de Métodos de Investigación y Diagnóstico en Educación de la Facultad de Ciencias de la Educación de la Universidad de Granada Sus principales líneas de investigación so la Orientación educativa y la calidad en educación. Su dirección postal es: Facultad de Ciencias de la Educación. Campus Universitario de Cartuja, s/n. C.P. 18071 - Granada (España).

\section{Zurita-Ortega, Félix (felixzo@ugr.es)}

Profesor Titular del Departamento de Didáctica de la Expresión Musical, Plástica y Corporal de la de la Facultad de Ciencias de la Educación de la Universidad de Granada. Su dirección postal es: Facultad de Ciencias de la Educación. Campus Universitario de Cartuja, s/n. C.P. 18071 - Granada (España).

\section{Castro-Sánchez, Manuel (mailto:mcastros@ual.es)}

Profesor del Departamento de Educación (área de Didáctica de la Expresión corporal) de la Universidad de Almería. Su dirección postal es Edificio Central. Ctra. Sacramento s/n. La Cañada de San Urbano 04120 Almería (España).
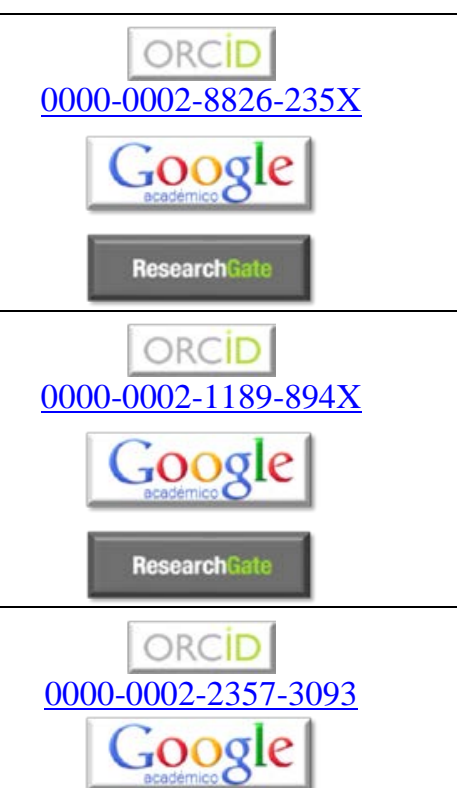

\section{Espejo-Garcés, Tamara (tamara.espejo@uca.es)}

Departamento de Didáctica y Organización escolar. Universidad de Cádiz. Su dirección postal es Facultad de Ciencias de la Educación. Campus Universitario Río San Pedro. Puerto Real (Cádiz).

\section{Chacón-Cuberos, Ramón (rchacón@ugr.es)}

Departamento de Didácticas Integradas. Universidad de Huelva. Su dirección postal es Facultad de Ciencias de la Educación. Avenida de las Fuerzas Armadas, S/N. 21071 Huelva (España) 
Martínez-Martínez, Asunción; Zurita-Ortega, Félix; Castro-Sánchez, Manuel; Espejo-Garcés, Tamara; ChacónCuberos, Ramón \& Ruiz-Rico-Ruiz, Gerardo (2017). La elección de la lengıa extraniera romo titılación universitaria: Expectativas y rasgos de estudio. RELIEVE, 23(2), art. 4. doi: http://doi.org/10.7203/relieve.23.2.8115

\title{
RELIEVE
}

\section{Revista ELectrónica de Investigación y EValuación Educativa E-Journal of Educational Research, Assessment and Evaluation}

[ISSN: 1134-4032]

\begin{abstract}
(C) Copyright, RELIEVE. Reproduction and distribution of this articles it is authorized if the content is no modified and their origin is indicated (RELIEVE Journal, volume, number and electronic address of the document).

(C) Copyright, RELIEVE. Se autoriza la reproducción y distribución de este artículo siempre que no se modifique el contenido y se indique su origen (RELIEVE, volumen, número y dirección electrónica del documento).
\end{abstract}

